MATHEMATICS OF COMPUTATION

Volume 69, Number 232, Pages 1505-1519

S 0025-5718(99)01176-X

Article electronically published on May 20, 1999

\title{
MONOTONICITY PRESERVATION ON TRIANGLES
}

\author{
MICHAEL S. FLOATER AND J. M. PEÑA
}

\begin{abstract}
It is well known that Bernstein polynomials on triangles preserve monotonicity. In this paper we define and study three kinds of monotonicity preservation of systems of bivariate functions on a triangle. We characterize and compare several of these systems and derive some geometric applications.
\end{abstract}

\section{INTRODUCTION}

In geometric modelling it is an advantage if the curve or surface being modelled tends to preserve the shape of its control polygon or control net respectively. For curves and surfaces which can be represented as graphs of univariate and bivariate functions respectively, one of the simplest shape properties is monotonicity.

Two recent papers (3] and [1]) present characterizations of systems of univariate functions that preserve monotonicity on an interval. Specifically, it was shown in Proposition 2.3 of [1] that a sequence of functions $u_{0}, u_{1}, \ldots, u_{n}$ defined on an interval $[a, b]$ is monotonicity preserving if and only if the function $\sum_{j=0}^{n} u_{j}(x)$ is constant and the function $\sum_{j=i}^{n} u_{j}(x)$ is increasing for $i=1, \ldots, n$. It was further shown in [1] how monotonicity preservation can be reformulated in terms of the positivity of minors of collocation matrices of $u_{0}, u_{1}, \ldots, u_{n}$, and that total positivity (see 11 and 9 ) is a sufficient condition for monotonicity preservation.

As far as we are aware the only known non-trivial basis of functions on a triangle which preserves monotonicity is the Bernstein basis of polynomials (see for instance Sec. 5.1 of [9]). In this paper we address two natural questions which arise from this: (1) is the Bernstein basis unique in having this property, and (2) if not, what characterizes such bases and what other shape properties do they possess? As we will see, the answers depend on how one defines monotonicity preservation.

In Section 2 we introduce notation and basic definitions, and derive some preliminary results. We define three types of systems of $\left(\begin{array}{c}n+2 \\ 2\end{array}\right)$ functions defined on a triangle: axially monotonicity preserving (AMP), monotonicity preserving (MP) and strongly monotonicity preserving (SMP) systems. They satisfy the relation

$$
\mathrm{SMP} \Rightarrow \mathrm{MP} \Rightarrow \mathrm{AMP} \text {. }
$$

In Section 3 we characterize AMP systems and from this derive two geometric properties of parametric surfaces generated by AMP systems (of nonnegative functions): the convex hull property and a length-diminishing property of iso-curves. It

Received by the editor May 27, 1997 and, in revised form, December 7, 1998.

1991 Mathematics Subject Classification. Primary 41A10, 65D17; Secondary 41 A63.

Key words and phrases. Monotonicity, shape preservation, bivariate Bernstein polynomials, control net.

The authors were supported in part by the EU project CHRX-CT94-0522. 
follows that the Bernstein basis is just one of a large class of function systems having desirable shape preserving properties when used to model surfaces in computer aided design.

The length-diminishing property of nonnegative AMP systems contrasts with the fact that a similar area-diminishing property does not hold even for Bézier surfaces, as pointed out by Goodman [9], p. 346. Some related variation diminishing properties of Bernstein polynomials on triangles have been studied in [7] and [8]. At the end of Section 3 we show that AMP systems also have the interesting property (distinct from the univariate case) of linearity preservation. Moreover, by a minor modification one can construct a new AMP system with the stronger property of linear precision.

In the remaining sections $(4,5$, and 6$)$ we compare AMP systems with MP and SMP systems for small $n$. We begin by showing in Section 4 that the case $n=1$ (three functions) is somewhat trivial, as the three classes of systems AMP, MP and SMP are then equivalent. Next, in Section 5, we focus on the non-trivial case $n=2$ and derive a more explicit characterization of AMP systems of six functions. Correspondingly, in Section 6 we characterize MP and SMP systems of six functions.

From the three characterizations in the case $n=2$, we make two interesting observations. The first is that AMP and MP systems are surprisingly closely related. The second is that, in contrast to AMP and MP systems, there are very few SMP systems. In fact, except and trivial cases, the only systems of six functions which are SMP are quadratic polynomials whose leading terms are a common multiple of the corresponding quadratic Bernstein polynomials. Thus strong monotonicity preservation is an example of a property which is unique to the Bernstein basis (and trivial extensions of it). This is not untypical of the Bernstein basis. For example, in the univariate case, the Bernstein basis is the one which has both optimal shape preserving properties [2] and optimal stability (among non-negative polynomials) [6] (see also [4]).

\section{BASIC DEFINITIONS AND PRELIMINARY RESULTS}

Let $\mathbf{i}=\left(i_{0}, i_{1}, i_{2}\right)$ denote a multi-index with $i_{0}, i_{1}, i_{2}$ in $\mathbb{Z}_{+}=\{0,1,2, \ldots\}$. We denote by $|\mathbf{i}|$ the sum of the coefficients $i_{0}+i_{1}+i_{2}$. We let $\mathbf{e}_{0}=(1,0,0)$, $\mathbf{e}_{1}=(0,1,0), \mathbf{e}_{2}=(0,0,1)$ and $\mathbf{f}_{0}=(0,1,1), \mathbf{f}_{1}=(1,0,1), \mathbf{f}_{2}=(1,1,0)$.

Let $p_{0}, p_{1}, p_{2}$ be three non-collinear points in $\mathbb{R}^{2}$ and let $T \subset \mathbb{R}^{2}$ be their convex hull, the triangle with vertices $p_{0}, p_{1}, p_{2}$. For some $n \geq 1$ suppose we are given, for each $\mathbf{i}$ such that $|\mathbf{i}|=n$, a function $\phi_{\mathbf{i}}: T \rightarrow \mathbb{R}$. There are $\left(\begin{array}{c}n+2 \\ 2\end{array}\right)$ such functions, and we will refer to them as a system and write $\left(\phi_{\mathbf{i}}\right)_{|\mathbf{i}|=n}$. Given also associated coefficients $c_{\mathbf{i}} \in \mathbb{R},|\mathbf{i}|=n$, we can define a function $f: T \rightarrow \mathbb{R}$ by

$$
f(x)=\sum_{|\mathbf{i}|=n} c_{\mathbf{i}} \phi_{\mathbf{i}}(x), \quad x \in T .
$$

Central to our discussion will be the notion of shape preservation; we are interested in systems $\left(\phi_{\mathbf{i}}\right)_{|\mathbf{i}|=n}$ for which $f$ in (2.1) tends to mimic the shape of its control net defined by the coefficients $c_{\mathbf{i}}$. In order to define the control net, consider the $\left(\begin{array}{c}n+2 \\ 2\end{array}\right)$ points in $T$,

$$
x_{\mathbf{i}}=\frac{i_{0}}{n} p_{0}+\frac{i_{1}}{n} p_{1}+\frac{i_{2}}{n} p_{2}, \quad|\mathbf{i}|=n .
$$




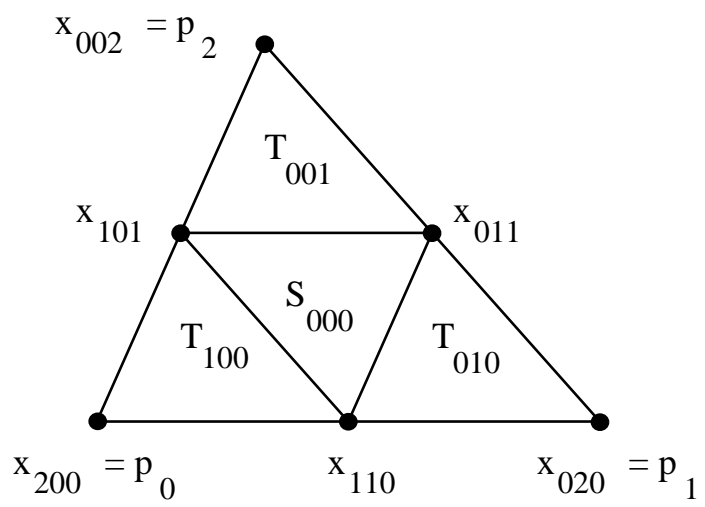

FIgURE 1. The triangulation in the case $n=2$

These points form the vertices of a natural triangulation of $T$ whose triangles are of two types. For $|\mathbf{i}|=n-1$, we let $T_{\mathbf{i}}$ be the triangle with vertices $x_{\mathbf{i}+\mathbf{e}_{0}}, x_{\mathbf{i}+\mathbf{e}_{1}}$, $x_{\mathbf{i}+\mathbf{e}_{2}}$, and for $|\mathbf{i}|=n-2$, we let $S_{\mathbf{i}}$ be the triangle with vertices $x_{\mathbf{i}+\mathbf{f}_{0}}, x_{\mathbf{i}+\mathbf{f}_{1}}, x_{\mathbf{i}+\mathbf{f}_{2}}$. Then

$$
\mathcal{T}=\left\{T_{\mathbf{i}}\right\}_{|\mathbf{i}|=n-1} \cup\left\{S_{\mathbf{i}}\right\}_{|\mathbf{i}|=n-2}
$$

is the regular triangulation of $T$ formed by those lines parallel to the sides of $T$ through the points $x_{\mathbf{i}}$ as illustrated in Figure 1. We define the control net of $f$ to be the function $p: T \rightarrow \mathbb{R}$ which is linear on each triangle in $\mathcal{T}$ and satisfies $p\left(x_{\mathbf{i}}\right)=c_{\mathbf{i}},|\mathbf{i}|=n$.

The particular kind of shape preservation we are interested in is monotonicity preservation.

Definition 2.1. Let $d$ be a vector in $\mathbb{R}^{2}$ with $d \neq 0$. We say that the system $\left(\phi_{\mathbf{i}}\right)_{|\mathbf{i}|=n}$ is monotonicity preserving with respect to $d$, if it has the property that $f$ in (2.1) is (monotonically) increasing in the direction $d$ whenever its control net $p$ is increasing in the direction $d$.

Let us remark that by replacing $f$ and $c_{\mathbf{i}}$ in $(2.1)$ by $-f$ and $-c_{\mathbf{i}}$ one can readily show that if the system $\left(\phi_{\mathbf{i}}\right)_{|\mathbf{i}|=n}$ is monotonicity preserving with respect to $d$, then it is also monotonicity preserving with respect to $-d$. Moreover, this observation leads us to a further one which will play an important role in the forthcoming discussion: a system which is monotonicity preserving with respect to $d$ has the property that if the control net of $f$ in (2.1) is constant in the direction $d$, then so is $f$.

Definition 2.2. We say that the system $\left(\phi_{\mathbf{i}}\right)_{|\mathbf{i}|=n}$ is monotonicity preserving (MP) if it is monotonicity preserving with respect to all vectors $d \in \mathbb{R}^{2}, d \neq 0$.

A system of functions on $T$ which will serve as a model of monotonicity preservation is the Bernstein basis. Given any point $x \in \mathbb{R}^{2}$, we identify it with its barycentric coordinates $\lambda_{0}(x), \lambda_{1}(x), \lambda_{2}(x)$ defined by

$$
\lambda_{0}(x) p_{0}+\lambda_{1}(x) p_{1}+\lambda_{2}(x) p_{2}=x
$$

and

$$
\lambda_{0}(x)+\lambda_{1}(x)+\lambda_{2}(x)=1 .
$$


The three functions $\lambda_{0}, \lambda_{1}, \lambda_{2}$ are linear on $\mathbb{R}^{2}$, and

$$
\left\{x \in \mathbb{R}^{2}: \lambda_{0}(x), \lambda_{1}(x), \lambda_{2}(x) \geq 0\right\}=T \quad \text { and } \quad \lambda_{j}\left(p_{i}\right)=\delta_{i j} .
$$

The Bernstein polynomials

$$
B_{\mathbf{i}}(x)=\frac{|\mathbf{i}| !}{i_{0} ! i_{1} ! i_{2} !} \lambda_{0}^{i_{0}}(x) \lambda_{1}^{i_{1}}(x) \lambda_{2}^{i_{2}}(x), \quad|\mathbf{i}|=n,
$$

form a basis for the linear space of polynomials of degree $\leq n$ (see Chapter 18 of [5] or Section 6.3 of [10]).

It seems to have been first suggested by Goodman 9 that Bernstein polynomials on triangles are monotonicity preserving. To understand this, let us consider the directional derivative $D_{d} f$ of a differentiable function $f: T \rightarrow \mathbb{R}$ in the direction $d=\left(d_{1}, d_{2}\right)$ at a point $x=\left(x_{1}, x_{2}\right)$ :

$$
D_{d} f(x)=d_{1} \frac{\partial f}{\partial x_{1}}(x)+d_{2} \frac{\partial f}{\partial x_{2}}(x) .
$$

The following result is well known (cf. Section 17.4 of [5]).

Lemma 2.3. For a given vector $d \in \mathbb{R}^{2}$, let $\gamma_{0}, \gamma_{1}, \gamma_{2}$ be the solutions of the equations

$$
\gamma_{0} p_{0}+\gamma_{1} p_{1}+\gamma_{2} p_{2}=d
$$

and

$$
\gamma_{0}+\gamma_{1}+\gamma_{2}=0
$$

If $f: T \rightarrow \mathbb{R}$ is such that

$$
f(x)=g\left(\lambda_{0}(x), \lambda_{1}(x), \lambda_{2}(x)\right)
$$

for some differentiable trivariate function $g: \mathbb{R}^{3} \rightarrow \mathbb{R}$, then

$$
D_{d} f=\gamma_{0} \frac{\partial g}{\partial \lambda_{0}}+\gamma_{1} \frac{\partial g}{\partial \lambda_{1}}+\gamma_{2} \frac{\partial g}{\partial \lambda_{2}} .
$$

Next we want to know how to establish the monotonicity of a control net in a direction $d$. As the following lemma will show, this can be done by checking the signs of the difference operators

$$
\begin{gathered}
E_{d} c_{\mathbf{i}}:=\gamma_{0} c_{\mathbf{i}+\mathbf{e}_{0}}+\gamma_{1} c_{\mathbf{i}+\mathbf{e}_{1}}+\gamma_{2} c_{\mathbf{i}+\mathbf{e}_{2}}, \quad|\mathbf{i}|=n-1, \\
F_{d} c_{\mathbf{i}}:=\left(\gamma_{1}+\gamma_{2}\right) c_{\mathbf{i}+\mathbf{f}_{0}}+\left(\gamma_{0}+\gamma_{2}\right) c_{\mathbf{i}+\mathbf{f}_{1}}+\left(\gamma_{0}+\gamma_{1}\right) c_{\mathbf{i}+\mathbf{f}_{2}}, \quad|\mathbf{i}|=n-2,
\end{gathered}
$$

which reflect the slopes of the control net over triangles of type $T_{\mathbf{i}}$ and $S_{\mathbf{i}}$ respectively.

Lemma 2.4. The control net $p: T \rightarrow \mathbb{R}$ is increasing in the direction $d \in \mathbb{R}^{2}$, $d \neq 0$, if and only if $E_{d} c_{\mathbf{i}} \geq 0$ for $|\mathbf{i}|=n-1$ and $F_{d} c_{\mathbf{i}} \geq 0$ for $|\mathbf{i}|=n-2$.

Proof. Let $F: T \rightarrow \mathbb{R}$ be the linear function

$$
F(x)=\lambda_{0}(x) a_{100}+\lambda_{1}(x) a_{010}+\lambda_{2}(x) a_{001} .
$$

If for given $\mathbf{i}=\left(i_{0}, i_{1}, i_{2}\right),|\mathbf{i}|=n-1$, we let $a_{\mathbf{e}_{k}}=n c_{\mathbf{i}+\mathbf{e}_{k}}-K, k=0,1,2$, and

$$
K=i_{0} c_{\mathbf{i}+\mathbf{e}_{0}}+i_{1} c_{\mathbf{i}+\mathbf{e}_{1}}+i_{2} c_{\mathbf{i}+\mathbf{e}_{2}},
$$


then a straightforward calculation using (2.2) shows that $F\left(x_{\mathbf{i}+\mathbf{e}_{k}}\right)=c_{\mathbf{i}+\mathbf{e}_{k}}, k=$ $0,1,2$, and so $\left.p\right|_{T_{\mathbf{i}}}=\left.F\right|_{T_{\mathbf{i}}}$. Therefore for $x \in \operatorname{Int}\left(T_{\mathbf{i}}\right)$ we find from Lemma 2.3 that

$$
D_{d} p(x)=D_{d} F(x)=E_{d} a_{\mathbf{0}}=n E_{d} c_{\mathbf{i}} .
$$

If for $|\mathbf{i}|=n-2$, we let $a_{100}=n\left(c_{\mathbf{i}+\mathbf{f}_{1}}+c_{\mathbf{i}+\mathbf{f}_{2}}\right)-K, a_{010}=n\left(c_{\mathbf{i}+\mathbf{f}_{2}}+c_{\mathbf{i}+\mathbf{f}_{0}}\right)-K$, $a_{001}=n\left(c_{\mathbf{i}+\mathbf{f}_{0}}+c_{\mathbf{i}+\mathbf{f}_{1}}\right)-K$, and

$$
K=\left(i_{0}+i_{1}+1\right) c_{\mathbf{i}+\mathbf{f}_{2}}+\left(i_{0}+i_{2}+1\right) c_{\mathbf{i}+\mathbf{f}_{1}}+\left(i_{1}+i_{2}+1\right) c_{\mathbf{i}+\mathbf{f}_{0}},
$$

then a further calculation using (2.2) shows that $F\left(x_{\mathbf{i}+\mathbf{f}_{k}}\right)=c_{\mathbf{i}+\mathbf{f}_{k}}, k=0,1,2$. Therefore $\left.p\right|_{S_{\mathbf{i}}}=\left.F\right|_{S_{\mathbf{i}}}$, and so, for $x \in \operatorname{Int}\left(S_{\mathbf{i}}\right)$,

$$
D_{d} p(x)=D_{d} F(x)=n F_{d} c_{\mathbf{i}}
$$

Returning now to the Bernstein case $\phi_{\mathbf{i}}=B_{\mathbf{i}}$ in (2.1), we use Lemma 2.3 and a change of summation index in order to derive the identity (see also Chapter 18 of [5])

$$
D_{d} f(x)=n \sum_{|\mathbf{i}|=n-1} E_{d} c_{\mathbf{i}} B_{\mathbf{i}}(x) .
$$

From Lemma 2.4 it therefore follows that the system $\left(B_{\mathbf{i}}\right)_{|\mathbf{i}|=n}$ is monotonicity preserving, confirming the assertion in [9]. In fact we notice that the expression for $D_{d} f(x)$ in (2.8) is independent of the differences $F_{d} c_{\mathbf{i}},|\mathbf{i}|=n-2$, and therefore independent of the gradient of the control net $p$ over triangles of type $S_{i}$. This suggests defining an evidently stronger property than monotonicity preservation which the Bernstein polynomials also satisfy:

Definition 2.5. We say that the system $\left(\phi_{\mathbf{i}}\right)_{|\mathbf{i}|=n}$ is strongly monotonicity preserving (SMP) provided that for all non-zero $d \in \mathbb{R}^{2}$, if $E_{d} c_{\mathbf{i}} \geq 0$ for $|\mathbf{i}|=n-1$, then $f$ in (2.1) is increasing in the direction $d$.

On the other hand, it seems to us worthwhile also to study a property which is clearly weaker than monotonicity preservation by restricting $d$ to the directions of the edges of the triangle $T$. Following the literature on convexity preservation (see e.g. [12]), we view the three edges of $T$ as 'axes' and define the property axial monotonicity preservation accordingly:

Definition 2.6. We say that the system $\left(\phi_{\mathbf{i}}\right)_{|\mathbf{i}|=n}$ is axially monotonicity preserving $(\mathrm{AMP})$ if it is monotonicity preserving with respect to the vectors $p_{1}-p_{0}, p_{2}-p_{1}$, and $p_{0}-p_{2}$.

We will now derive some basic properties of AMP systems and consequently also of MP and SMP systems.

Lemma 2.7. Let $\left(\phi_{\mathbf{i}}\right)_{|\mathbf{i}|=n}$ be an AMP system and let $f$ be the function in (2.1). If $c_{\mathbf{i}}=c_{\mathbf{j}}$ whenever $i_{0}=j_{0}$ (resp. $i_{1}=j_{1}, i_{2}=j_{2}$ ), then $f$ is constant in the direction $p_{2}-p_{1}$ (resp. $\left.p_{0}-p_{2}, p_{1}-p_{0}\right)$.

Proof. If $d=p_{2}-p_{1}$, then from (2.5) and (2.6) we have $\gamma_{0}=0, \gamma_{1}=-1$, and $\gamma_{2}=1$. It follows that

$$
E_{d} c_{\mathbf{i}}=c_{\mathbf{i}+\mathbf{e}_{2}}-c_{\mathbf{i}+\mathbf{e}_{1}}, \quad|\mathbf{i}|=n-1, \quad \text { and } \quad F_{d} c_{\mathbf{i}}=c_{\mathbf{i}+\mathbf{f}_{1}}-c_{\mathbf{i}+\mathbf{f}_{2}}, \quad|\mathbf{i}|=n-2 .
$$

Thus the control net of $f$ is constant in the direction $p_{2}-p_{1}$ if and only if $c_{\mathbf{i}+\mathbf{e}_{2}}=$ $c_{\mathbf{i}+\mathbf{e}_{1}}$ for $|\mathbf{i}|=n-1$. Therefore if $c_{\mathbf{i}}=c_{\mathbf{j}}$ whenever $i_{0}=j_{0}$, the control net of $f$ is 
constant in the direction $p_{2}-p_{1}$, and since $\left(\phi_{\mathbf{i}}\right)_{|\mathbf{i}|=n}$ is AMP, $f$ is also constant in the direction $p_{2}-p_{1}$. The remaining two cases can be treated analogously.

A simple consequence of Lemma 2.7 is the following.

Proposition 2.8. If $\left(\phi_{\mathbf{i}}\right)_{|\mathbf{i}|=n}$ is an AMP system, then $\sum_{|\mathbf{i}|=n} \phi_{\mathbf{i}}$ is a constant function.

Proof. Letting $c_{\mathbf{i}}=1$ in Lemma 2.7, we deduce that $\sum_{|\mathbf{i}|=n} \phi_{\mathbf{i}}$ is constant in the two linearly independent directions $p_{2}-p_{1}$ and $p_{0}-p_{2}$.

The following necessary conditions are also consequences of Lemma 2.7.

Proposition 2.9. Let $\left(\phi_{\mathbf{i}}\right)_{|\mathbf{i}|=n}$ be an AMP system. Then:

(i) For all $i_{0}=0,1, \ldots, n$, the function $\sum_{|\mathbf{j}|=n, j_{0}=i_{0}} \phi_{\mathbf{j}}$ is constant in the direction $p_{2}-p_{1}$.

(ii) For all $i_{1}=0,1, \ldots, n$, the function $\sum_{|\mathbf{j}|=n, j_{1}=i_{1}} \phi_{\mathbf{j}}$ is constant in the direction $p_{0}-p_{2}$.

(iii) For all $i_{2}=0,1, \ldots, n$, the function $\sum_{|\mathbf{j}|=n, j_{2}=i_{2}} \phi_{\mathbf{j}}$ is constant in the direction $p_{1}-p_{0}$.

Proof. In case (i) we let $c_{\mathbf{j}}=1$ when $j_{0}=i_{0}$ and $c_{\mathbf{j}}=0$ otherwise. We then apply Lemma 2.7 to $f(x)=\sum_{|\mathbf{j}|=n, j_{0}=i_{0}} \phi_{\mathbf{j}}$. Cases (ii) and (iii) are similar.

In particular we observe that if $\left(\phi_{\mathbf{i}}\right)_{|\mathbf{i}|=n}$ is axially monotonicity preserving, then $\phi_{n 00}, \phi_{0 n 0}$, and $\phi_{00 n}$ are essentially univariate functions, and so all the three types of monotonicity preservation in Definitions 2.2, 2.5, 2.6 place heavy restrictions on the functions in the system, unlike in the univariate case.

\section{Axially monotonicity PRESERVING Systems}

In this section we characterize all axially monotonicity preserving systems $\left(\phi_{\mathbf{i}}\right)_{|\mathbf{i}|=n}$ and derive some geometric properties of them.

We begin with the characterization and, to this end, we define the functions

$$
\psi_{\mathbf{i}}^{0}=\sum_{j=0}^{i_{1}} \phi_{i_{0}, j, n-i_{0}-j}, \quad \psi_{\mathbf{i}}^{1}=\sum_{j=0}^{i_{2}} \phi_{n-i_{1}-j, i_{1}, j}, \quad \psi_{\mathbf{i}}^{2}=\sum_{j=0}^{i_{0}} \phi_{j, n-i_{2}-j, i_{2}},
$$

for $|\mathbf{i}|=n$, which are partial sums of rows of the $\phi_{\mathbf{i}}$.

Theorem 3.1. Let $\left(\phi_{\mathbf{i}}\right)_{|\mathbf{i}|=n}$ be a system of $\left(\begin{array}{c}n+2 \\ 2\end{array}\right)$ functions. Then the following properties are equivalent:

(i) $\left(\phi_{\mathbf{i}}\right)_{|\mathbf{i}|=n}$ is $A M P$.

(ii) For all $\mathbf{i}$ with $|\mathbf{i}|=n$, and $k=0,1,2$, the function $\psi_{\mathbf{i}}^{k}$ is constant in the direction $d=p_{k+2}-p_{k+1}$ when $i_{k+2}=0$, and increasing in the direction $d$ when $i_{k+2}>0($ and $k+j$ denotes $(k+j) \bmod 3$ for $j \in \mathbb{Z})$.

Proof. Suppose that $\left(\phi_{\mathbf{i}}\right)_{|\mathbf{i}|=n}$ is AMP. Then Proposition 2.9 shows that for $i_{0}=$ $0,1, \ldots, n$, the function $\psi_{i_{0}, n-i_{0}, 0}^{0}$ is constant in the direction $p_{2}-p_{1}$. If for any $\mathbf{i}=\left(i_{0}, i_{1}, i_{2}\right),|\mathbf{i}|=n$, such that $i_{2}>0$, we let $c_{i_{0}, j, n-i_{0}-j}=1$ for $j=0,1, \ldots, i_{1}$ and $c_{\mathbf{i}}=0$ otherwise, then the control net $p$ is increasing in the direction $p_{2}-p_{1}$, and therefore $\psi_{\mathbf{i}}^{0}$ is increasing in the direction $p_{2}-p_{1}$. Since the cases $k=1,2$ are similar, we have established property (ii). 
Next suppose that the system $\left(\phi_{\mathbf{i}}\right)_{|\mathbf{i}|=n}$ satisfies (ii). By comparing coefficients, one can show that for $x \in T$ and $i_{0} \in\{0,1, \ldots, n\}$ we have

$$
\begin{aligned}
& \sum_{i_{1}=0}^{n-i_{0}} c_{i_{0}, i_{1}, n-i_{0}-i_{1}} \phi_{i_{0}, i_{1}, n-i_{0}-i_{1}} \\
& \quad=c_{i_{0}, n-i_{0}, 0} \psi_{i_{0}, n-i_{0}, 0}^{0}+\sum_{i_{1}=0}^{n-i_{0}-1}\left(c_{i_{0}, i_{1}, n-i_{0}-i_{1}}-c_{i_{0}, i_{1}+1, n-i_{0}-i_{1}-1}\right) \psi_{i_{0}, i_{1}, n-i_{0}-i_{1}}^{0} .
\end{aligned}
$$

If $x_{1}, x_{2}$ are two distinct points in $T$ and the vector $x_{2}-x_{1}$ has the same direction as $p_{2}-p_{1}$, we also have, from (ii),

$$
\psi_{i_{0}, n-i_{0}, 0}^{0}\left(x_{1}\right)=\psi_{i_{0}, n-i_{0}, 0}^{0}\left(x_{2}\right)
$$

and hence

$$
\begin{aligned}
f\left(x_{2}\right)- & f\left(x_{1}\right) \\
= & \sum_{i_{0}=0}^{n} \sum_{i_{1}=0}^{n-i_{0}} c_{i_{0}, i_{1}, n-i_{0}-i_{1}} \phi_{i_{0}, i_{1}, n-i_{0}-i_{1}}\left(x_{2}\right) \\
& +\sum_{i_{0}=0}^{n} \sum_{i_{1}=0}^{n-i_{0}} c_{i_{0}, i_{1}, n-i_{0}-i_{1}} \phi_{i_{0}, i_{1}, n-i_{0}-i_{1}}\left(x_{1}\right) \\
= & \sum_{i_{0}=0}^{n} \sum_{i_{1}=0}^{n-i_{0}}\left(c_{i_{0}, i_{1}, n-i_{0}-i_{1}}-c_{i_{0}, i_{1}+1, n-i_{0}-i_{1}-1}\right) \\
& \times\left(\psi_{i_{0}, i_{1}, n-i_{0}-i_{1}}^{0}\left(x_{2}\right)-\psi_{i_{0}, i_{1}, n-i_{0}-i_{1}}^{0}\left(x_{1}\right)\right) \\
= & \sum_{|\mathbf{i}|=n-1} E_{p_{2}-p_{1}} c_{\mathbf{i}}\left(\psi_{\mathbf{i}+\mathbf{e}_{2}}^{0}\left(x_{2}\right)-\psi_{\mathbf{i}+\mathbf{e}_{2}}^{0}\left(x_{1}\right)\right) .
\end{aligned}
$$

This means that, again by (ii), if the control net of $f$ is increasing in the direction $d=p_{2}-p_{1}$, then so is $f$. An identical argument for the other two axial directions establishes that the system $\left(\phi_{\mathbf{i}}\right)_{|\mathbf{i}|=n}$ is AMP.

As we would expect, Theorem 3.1 can be used as an alternative way to show that the Bernstein polynomials are AMP. Indeed, a straightforward calculation using Lemma 2.3 shows that

$$
\sum_{i_{1}=0}^{n-i_{0}} B_{i_{0}, i_{1}, n-i_{0}-i_{1}}(x)=\left(\begin{array}{c}
n \\
i_{0}
\end{array}\right) \lambda_{0}^{i_{0}}(x)\left(1-\lambda_{0}(x)\right)^{n-i_{0}},
$$

which is constant in the direction $p_{2}-p_{1}$, and using the identity (see Chapter 18 of [5])

$$
D_{d} B_{\mathbf{i}}(x)=n\left(\gamma_{0} B_{\mathbf{i}-\mathbf{e}_{0}}(x)+\gamma_{1} B_{\mathbf{i}-\mathbf{e}_{1}}(x)+\gamma_{2} B_{\mathbf{i}-\mathbf{e}_{2}}(x)\right),
$$

one can show that

$$
D_{p_{2}-p_{1}} \psi_{\mathbf{i}}^{0}(x)=n B_{\mathbf{i}-\mathbf{e}_{2}}(x) \geq 0 .
$$


In the remainder of this section we explore some geometric properties of axially monotonicity preserving systems.

Given an AMP system $\left(\phi_{\mathbf{i}}\right)_{|\mathbf{i}|=n}$ of nonnegative functions, not all of which are zero, Proposition 2.8 implies that the functions sum up to a positive constant. Thus, by merely dividing them by this constant, we obtain a new AMP system which is also a blending system (also called a partition of unity), that is, $\phi_{\mathbf{i}} \geq 0$ and $\sum_{|\mathbf{i}|=n} \phi_{\mathbf{i}}(x)=1$, for all $x \in T$. Blending systems have two important aspects. One is that since the functions sum to one, for a given set of points $\left(C_{\mathbf{i}}\right)_{|\mathbf{i}|=n}, C_{\mathbf{i}} \in \mathbb{R}^{3}$, we can take a corresponding affine combination in order to define a parametric surface $S: T \rightarrow \mathbb{R}^{3}$ by

$$
S(x)=\sum_{|\mathbf{i}|=n} \phi_{\mathbf{i}}(x) C_{\mathbf{i}}, \quad x \in T .
$$

In keeping with the Bernstein case $\phi_{\mathbf{i}}=B_{\mathbf{i}}$, we will refer to the $C_{\mathbf{i}}$ as the control points of $S$. In the Bernstein case, the surface $S$ is called a Bézier surface (see Chapter 18 of [5]).

The second aspect of blending systems is that since the functions are nonnegative as well as summing to one, they have the convex hull property, i.e. any point of the surface $S$ in (3.2) lies in the convex hull of its control points $C_{\mathbf{i}}$. This shape property gives a designer control over the rough shape of $S$.

Next we derive a shape property of AMP blending systems. For $\alpha \in[0,1)$ the two points $x_{\alpha}^{k}=(1-\alpha) p_{k}+\alpha p_{0}, k=1,2$, define a line segment $x_{\alpha}:[0,1] \rightarrow T$ given by

$$
x_{\alpha}(t)=(1-t) x_{\alpha}^{1}+t x_{\alpha}^{2}
$$

Let $\gamma_{\alpha}:[0,1] \rightarrow \mathbb{R}^{3}$ be the curve $\gamma_{\alpha}(t)=S\left(x_{\alpha}(t)\right)$, which we call an iso-curve of $S$, for the function $\lambda_{0}\left(x_{\alpha}(t)\right)$ is constant in $t$. For $j=0,1, \ldots, n-1$ let $P_{j}$ denote the polygonal arc with vertices $C_{j, n-j, 0}, C_{j, n-j-1,1}, \ldots, C_{j, 0, n-j}$. Let us call $P_{j}$ an iso-polygon, for its vertices are those control points $C_{i_{0}, i_{1}, i_{2}}, i_{0}+i_{1}+i_{2}=n$, whose multi-indices share the same first coordinate, namely $i_{0}=j$.

Let us recall that the total variation of a vector valued function $\gamma:[a, b] \rightarrow \mathbb{R}^{3}$ is defined by

$$
\operatorname{TV}(\gamma):=\sup _{\substack{t_{0}<\cdots<t_{N} \in[a, b] \\ N \geq 1}} \sum_{k=1}^{N}\left\|\gamma\left(t_{k}\right)-\gamma\left(t_{k-1}\right)\right\|,
$$

where $\|\cdot\|$ is any norm in $\mathbb{R}^{3}$. If $\operatorname{TV}(\gamma)<\infty$, then $\gamma$ is said to be a vector valued function of bounded variation. If $\|\cdot\|$ is the Euclidean norm, then $\operatorname{TV}(\gamma)$ is precisely the length $L(\gamma)$ of the curve. In the following we show that the length of any iso-curve of $S$ is bounded by the maximum length of its iso-polygons.

Proposition 3.2. Let $\left(\phi_{\mathbf{i}}\right)_{|\mathbf{i}|=n}$ be an AMP blending system. Then for $\alpha \in[0,1)$,

$$
L\left(\gamma_{\alpha}\right) \leq \max _{j=0,1, \ldots, n-1} L\left(P_{j}\right)
$$


Proof. For $N \geq 1,0 \leq t_{0}<t_{1}<\ldots<t_{N} \leq 1$ and $\alpha \in[0,1)$ we have, from equation (3.1),

$$
\begin{aligned}
& \sum_{k=1}^{N}\left\|\gamma_{\alpha}\left(t_{k}\right)-\gamma_{\alpha}\left(t_{k-1}\right)\right\| \\
& =\sum_{k=1}^{N}\left\|\sum_{|\mathbf{i}|=n-1} E_{p_{2}-p_{1}} C_{\mathbf{i}}\left(\psi_{\mathbf{i}+\mathbf{e}_{2}}^{0}\left(x_{\alpha}\left(t_{k}\right)\right)-\psi_{\mathbf{i}+\mathbf{e}_{2}}^{0}\left(x_{\alpha}\left(t_{k-1}\right)\right)\right)\right\| \\
& \leq \sum_{|\mathbf{i}|=n-1}\left\|E_{p_{2}-p_{1}} C_{\mathbf{i}}\right\| \sum_{k=1}^{N}\left(\psi_{\mathbf{i}+\mathbf{e}_{2}}^{0}\left(x_{\alpha}\left(t_{k}\right)\right)-\psi_{\mathbf{i}+\mathbf{e}_{2}}^{0}\left(x_{\alpha}\left(t_{k-1}\right)\right)\right) \\
& \leq \sum_{|\mathbf{i}|=n-1}\left\|E_{p_{2}-p_{1}} C_{\mathbf{i}}\right\| \psi_{\mathbf{i}+\mathbf{e}_{2}}^{0}\left(x_{\alpha}\left(t_{N}\right)\right) \\
& =\sum_{i_{0}=0}^{n} \sum_{i_{1}=0}^{n-i_{0}}\left\|C_{i_{0}, i_{1}, n-i_{0}-i_{1}}-C_{i_{0}, i_{1}+1, n-i_{0}-i_{1}-1}\right\| \psi_{i_{0}, i_{1}, n-i_{0}-i_{1}}^{0}\left(x_{\alpha}\left(t_{N}\right)\right) \\
& \leq \sum_{i_{0}=0}^{n} \psi_{i_{0}, n-i_{0}, 0}^{0}\left(x_{\alpha}\left(t_{N}\right)\right) L\left(P_{i_{0}}\right) \\
& \leq \sum_{|\mathbf{i}|=n} \phi_{\mathbf{i}}\left(x_{\alpha}\left(t_{N}\right)\right) \max _{i_{0}=0,1, \ldots, n-1} L\left(P_{i_{0}}\right)=\max _{i_{0}=0,1, \ldots, n-1} L\left(P_{i_{0}}\right) .
\end{aligned}
$$

Taking the supremum over all sequences satisfying $0 \leq t_{0}<t_{1}<\ldots<t_{N} \leq 1$, the result follows.

There is clearly an analogous property for iso-curves in the other two axial directions.

Proposition 3.2 implies that the iso-curves of a Bézier surface are not longer than the longest of its iso-polygons in the same direction. This is interesting in view of the surprising fact, established by Goodman (see p. 346 of [9]), that the surface area of a Bézier surface is not necessarily bounded above by the surface area of its control net.

Though Theorem 3.1 provides necessary and sufficient conditions for axial monotonicity preservation, it does not reveal a further necessary condition when the functions are differentiable, namely preservation of linearity, which is interesting in the light of the univariate case, in which there is no such property.

Theorem 3.3. Let $\left(\phi_{\mathbf{i}}\right)_{|\mathbf{i}|=n}$ be an AMP system of differentiable functions. Then if the control net $p$ of $f$ in (2.1) is linear, then so is $f$.

Proof. If $p$ is linear, we must have

$$
c_{\mathbf{i}}=\frac{i_{0}}{n} c_{n 00}+\frac{i_{1}}{n} c_{0 n 0}+\frac{i_{2}}{n} c_{00 n}, \quad|\mathbf{i}|=n .
$$

Therefore if we define the three functions on $T$,

$$
f_{k}(x)=\sum_{|\mathbf{i}|=n} \frac{i_{k}}{n} \phi_{\mathbf{i}}(x), \quad k=0,1,2,
$$


we find that

$$
f(x)=\sum_{|\mathbf{i}|=n} c_{\mathbf{i}} \phi(x)=f_{0}(x) c_{n 00}+f_{1}(x) c_{0 n 0}+f_{2}(x) c_{00 n} .
$$

As will be shown in Lemma 3.4 , the three functions $f_{0}, f_{1}, f_{2}$ are linear, and therefore $f$ is linear.

Lemma 3.4. Let $\left(\phi_{\mathbf{i}}\right)_{|\mathbf{i}|=n}$ be an AMP system of differentiable functions. Then there exist constants $a, b_{0}, b_{1}, b_{2} \in \mathbb{R}$ with $a \geq 0$ such that the functions defined in (3.4) satisfy

$$
f_{0}(x)=a \lambda_{0}(x)+b_{0}, \quad f_{1}(x)=a \lambda_{1}(x)+b_{1}, \quad f_{2}(x)=a \lambda_{2}(x)+b_{2} .
$$

Proof. Summing the functions $f_{k}$ and recalling Proposition 2.8, we have

$$
f_{0}(x)+f_{1}(x)+f_{2}(x)=\sum_{|\mathbf{i}|=n} \phi_{\mathbf{i}}(x)=K
$$

for some constant $K$ independent of $x$. Further, we have from Proposition 2.9 that $f_{0}$ is constant in the direction $p_{2}-p_{1}$, and so $f_{0}(x)=g_{0}\left(\lambda_{0}(x)\right)$ for some differentiable function $g_{0}:[0,1] \rightarrow \mathbb{R}$. Similarly there exist functions $g_{1}, g_{2}:[0,1] \rightarrow$ $\mathbb{R}$ such that $f_{1}(x)=g_{1}\left(\lambda_{1}(x)\right)$ and $f_{2}(x)=g_{2}\left(\lambda_{2}(x)\right)$, and then

$$
g_{0}\left(\lambda_{0}(x)\right)+g_{1}\left(\lambda_{1}(x)\right)+g_{2}\left(\lambda_{2}(x)\right)=K .
$$

Differentiating with respect to any $d \in \mathbb{R}^{2}$ and using Lemma 2.3, we find that

$$
\gamma_{0} g_{0}^{\prime}\left(\lambda_{0}(x)\right)+\gamma_{1} g_{1}^{\prime}\left(\lambda_{1}(x)\right)+\gamma_{2} g_{2}^{\prime}\left(\lambda_{2}(x)\right)=0,
$$

and choosing $\left(\gamma_{0}, \gamma_{1}, \gamma_{2}\right)$ to be $(1,-1,0)$ we deduce that

$$
g_{0}^{\prime}\left(\lambda_{0}(x)\right)=g_{1}^{\prime}\left(\lambda_{1}(x)\right) .
$$

Varying $x$ so that $\lambda_{0}(x)$ varies while $\lambda_{1}(x)$ remains constant, it follows that $g_{0}^{\prime}$ must be a constant function on $[0,1]$. Similarly, $g_{1}^{\prime}$ and $g_{2}^{\prime}$ must be constant, and moreover

$$
g_{0}^{\prime}\left(\lambda_{0}\right)=g_{1}^{\prime}\left(\lambda_{1}\right)=g_{2}^{\prime}\left(\lambda_{2}\right)=a,
$$

for some constant $a$. Integrating, we find that

$$
g_{0}\left(\lambda_{0}\right)=a \lambda_{0}+b_{0}, \quad g_{1}\left(\lambda_{1}\right)=a \lambda_{1}+b_{1}, \quad g_{2}\left(\lambda_{2}\right)=a \lambda_{2}+b_{2},
$$

for some constants $b_{0}, b_{1}, b_{2}$ (and summing the $g_{k}$ we have $K=a+b_{0}+b_{1}+b_{2}$ ).

If now the control net $p$ of $f$ is linear, then the coefficients $c_{\mathbf{i}}$ satisfy (3.3) and, from (3.5), $f$ takes the form

$$
f(x)=\left(a \lambda_{0}(x)+b_{0}\right) c_{n 00}+\left(a \lambda_{1}(x)+b_{1}\right) c_{0 n 0}+\left(a \lambda_{2}(x)+b_{2}\right) c_{00 n} .
$$

Therefore for any vector $d \in \mathbb{R}^{2}$,

$$
D_{d} f(x)=a\left(\gamma_{0} c_{n 00}+\gamma_{1} c_{0 n 0}+\gamma_{2} c_{00 n}\right)=a D_{d} p(x),
$$

and we see that because $\left(\phi_{\mathbf{i}}\right)_{|\mathbf{i}|=n}$ is AMP, we must have $a \geq 0$.

If $a=1$ and $b_{0}=b_{1}=b_{2}=0$ in Lemma 3.4, then the AMP system $\left(\phi_{\mathbf{i}}\right)_{|\mathbf{i}|=n}$ has linear precision. In other words, if $l: T \rightarrow \mathbb{R}$ is any linear function, then

$$
l(x)=\sum_{|\mathbf{i}|=n} l\left(x_{\mathbf{i}}\right) \phi_{\mathbf{i}}(x), \quad x \in T .
$$


Indeed, from (3.6) we have in this case that $f_{k}(x)=\lambda_{k}(x), k=0,1,2$. If we let $c_{\mathbf{i}}=l\left(x_{\mathbf{i}}\right)$, then (3.3) holds, and so from (3.5) we obtain

$$
\sum_{|\mathbf{i}|=n} l\left(x_{\mathbf{i}}\right) \phi_{\mathbf{i}}(x)=\sum_{|\mathbf{i}|=n} c_{\mathbf{i}} \phi_{\mathbf{i}}(x)=\lambda_{0}(x) c_{n 00}+\lambda_{1}(x) c_{0 n 0}+\lambda_{2}(x) c_{00 n}=l(x) .
$$

Given an arbitrary AMP system $\left(\phi_{\mathbf{i}}\right)_{|\mathbf{i}|=n}$, we can construct a new system $\left(\hat{\phi}_{\mathbf{i}}\right)_{|\mathbf{i}|=n}$ which is AMP and has linear precision. We can compute $a, b_{0}, b_{1}, b_{2}$ from $f_{0}, f_{1}, f_{2}$ and, if $a$ is strictly positive, one could for example define

$$
\hat{\phi}_{n 00}=\frac{1}{a}\left(\phi_{n 00}-b_{0}\right), \quad \hat{\phi}_{0 n 0}=\frac{1}{a}\left(\phi_{0 n 0}-b_{1}\right), \quad \hat{\phi}_{00 n}=\frac{1}{a}\left(\phi_{00 n}-b_{2}\right),
$$

and

$$
\hat{\phi}_{\mathbf{i}}=\frac{1}{a} \phi_{\mathbf{i}}
$$

for all other $\mathbf{i},|\mathbf{i}|=n$.

If the system $\left(\phi_{\mathbf{i}}\right)_{|\mathbf{i}|=n}$ has linear precision, then we remark that the graph of $f$ in (2.1) can be represented as a parametric surface $S$ of the form (3.2), as is well known for Bernstein polynomials. Indeed, we let $C_{\mathbf{i}}=\left(x_{\mathbf{i}}, c_{\mathbf{i}}\right)$, and then

$$
(x, f(x))=S(x), \quad x \in T .
$$

\section{AMP, MP AND SMP SYSTEMS WHEN $n=1$}

In this section we characterize all systems of differentiable functions $\left(\phi_{\mathbf{i}}\right)_{|\mathbf{i}|=n}$ satisfying Definitions 2.2, 2.5, 2.6 for the case $n=1$. We show that all such systems consist of linear functions, unlike in the univariate case.

Theorem 4.1. Let $\left(\phi_{\mathbf{i}}\right)_{|\mathbf{i}|=1}$ be a system of three differentiable functions. Then the following statements are equivalent:

(i) $\left(\phi_{\mathbf{i}}\right)_{|\mathbf{i}|=1}$ is $S M P$.

(ii) $\left(\phi_{\mathbf{i}}\right)_{|\mathbf{i}|=1}$ is $M P$.

(iii) $\left(\phi_{\mathbf{i}}\right)_{|\mathbf{i}|=1}$ is $A M P$.

(iv) There exist constants $a, b_{0}, b_{1}, b_{2} \in \mathbb{R}$ with $a \geq 0$ such that

$$
\phi_{100}(x)=a \lambda_{0}(x)+b_{0}, \quad \phi_{010}(x)=a \lambda_{1}(x)+b_{1}, \quad \phi_{001}(x)=a \lambda_{2}(x)+b_{2} .
$$

Proof. We observed earlier that (i) implies (ii) and (ii) implies (iii). Since the functions $f_{0}, f_{1}, f_{2}$ in (3.4) are precisely $\phi_{100}, \phi_{010}, \phi_{001}$ respectively, Lemma 3.4 shows that (iii) implies (iv). If (iv) holds, then $f$ in (2.1) takes the form

$$
f(x)=\left(a \lambda_{0}(x)+b_{0}\right) c_{100}+\left(a \lambda_{1}(x)+b_{1}\right) c_{010}+\left(a \lambda_{2}(x)+b_{2}\right) c_{001} .
$$

Therefore, for any vector $d \in \mathbb{R}^{2}$,

$$
D_{d} f(x)=a\left(\gamma_{0} c_{100}+\gamma_{1} c_{010}+\gamma_{2} c_{001}\right)=a E_{d} c_{0} .
$$

So from Definition 2.5, we deduce that the system $\left(\phi_{\mathbf{i}}\right)_{|\mathbf{i}|=1}$ is SMP.

As we noticed before, Bernstein polynomials are strongly monotonicity preserving. In the case $n=1$, we can confirm that they belong to the class of functions of type (iv) in Theorem 4.1 by setting $a=1$ and $b_{0}=b_{1}=b_{2}=0$. We remark also that in the case $n=1$, it is not surprising that monotonicity preservation and strong monotonicity preservation are equivalent since then the triangulation $\mathcal{T}=\left\{T_{000}\right\}$ contains no triangles of type $S_{\mathbf{i}}$. 


\section{AMP SYSTEMS WHEN $n=2$}

In Section 3 we characterized AMP systems in terms of the partial sums $\psi_{\mathbf{i}}$. In this section we derive a more explicit characterization in the case $n=2$ which expresses the six functions $\phi_{\mathbf{i}}$ as sums of univariate functions of barycentric coordinates.

Theorem 5.1. Let $\left(\phi_{\mathbf{i}}\right)_{|\mathbf{i}|=2}$ be a system of six differentiable functions. Then the following two properties are equivalent:

(i) $\left(\phi_{\mathbf{i}}\right)_{|\mathbf{i}|=2}$ is $A M P$.

(ii) There exist constants $a, b_{0}, b_{1}, b_{2} \in \mathbb{R}$ where $a \geq 0$, and there exist functions $g_{0}, g_{1}, g_{2}:[0,1] \rightarrow \mathbb{R}$ where $g_{k}^{\prime}(s) \leq a$ for $s \in[0,1], k=0,1,2$ and

$$
g_{0}^{\prime}(s)+g_{1}^{\prime}(t) \geq 0, \quad g_{1}^{\prime}(s)+g_{2}^{\prime}(t) \geq 0, \quad g_{2}^{\prime}(s)+g_{0}^{\prime}(t) \geq 0, \quad s, t \geq 0, \quad s+t \leq 1,
$$

such that

$$
\begin{aligned}
& \phi_{110}(x)=g_{0}\left(\lambda_{0}(x)\right)+g_{1}\left(\lambda_{1}(x)\right)-g_{2}\left(\lambda_{2}(x)\right), \\
& \phi_{101}(x)=g_{0}\left(\lambda_{0}(x)\right)-g_{1}\left(\lambda_{1}(x)\right)+g_{2}\left(\lambda_{2}(x)\right), \\
& \phi_{011}(x)=-g_{0}\left(\lambda_{0}(x)\right)+g_{1}\left(\lambda_{1}(x)\right)+g_{2}\left(\lambda_{2}(x)\right),
\end{aligned}
$$

and

$$
\begin{aligned}
& \phi_{200}(x)=a \lambda_{0}(x)+b_{0}-g_{0}\left(\lambda_{0}(x)\right), \\
& \phi_{020}(x)=a \lambda_{1}(x)+b_{1}-g_{1}\left(\lambda_{1}(x)\right), \\
& \phi_{002}(x)=a \lambda_{2}(x)+b_{2}-g_{2}\left(\lambda_{2}(x)\right) .
\end{aligned}
$$

Proof. Suppose that $\left(\phi_{\mathbf{i}}\right)_{|\mathbf{i}|=2}$ is axially monotonicity preserving. Then, by Lemma 2.7 , it can be deduced that there exist functions $g_{0}, g_{1}, g_{2}:[0,1] \rightarrow \mathbb{R}$ such that

$$
\begin{aligned}
& \left(\phi_{110}(x)+\phi_{101}(x)\right) / 2=g_{0}\left(\lambda_{0}(x)\right), \\
& \left(\phi_{010}(x)+\phi_{011}(x)\right) / 2=g_{1}\left(\lambda_{1}(x)\right), \\
& \left(\phi_{011}(x)+\phi_{101}(x)\right) / 2=g_{2}\left(\lambda_{2}(x)\right) .
\end{aligned}
$$

Taking linear combinations of these equations, we obtain equations (5.2). Further, by Lemma 3.4 there exist constants $a, b_{0}, b_{1}, b_{2} \in \mathbb{R}$ with $a \geq 0$ such that

$$
\begin{aligned}
& \phi_{200}(x)+\left(\phi_{110}(x)+\phi_{101}(x)\right) / 2=f_{0}(x)=a \lambda_{0}(x)+b_{0}, \\
& \phi_{020}(x)+\left(\phi_{110}(x)+\phi_{011}(x)\right) / 2=f_{1}(x)=a \lambda_{1}(x)+b_{1}, \\
& \phi_{002}(x)+\left(\phi_{101}(x)+\phi_{011}(x)\right) / 2=f_{2}(x)=a \lambda_{2}(x)+b_{2},
\end{aligned}
$$

and so we obtain equations (5.3). Substituting the expressions for the $\phi_{\mathbf{i}}$ in (5.2) and (5.3) into $f$ in (2.1) and employing Lemma 2.3, we find that for any vector $d \in \mathbb{R}^{2}$,

$$
\begin{aligned}
D_{d} f(x)= & \left(\left(a-g_{0}^{\prime}\left(\lambda_{0}(x)\right)\right) c_{200}+g_{0}^{\prime}\left(\lambda_{0}(x)\right)\left(c_{110}+c_{101}-c_{011}\right)\right) \gamma_{0} \\
& +\left(\left(a-g_{1}^{\prime}\left(\lambda_{1}(x)\right)\right) c_{020}+g_{1}^{\prime}\left(\lambda_{1}(x)\right)\left(c_{110}-c_{101}+c_{011}\right)\right) \gamma_{1} \\
& +\left(\left(a-g_{2}^{\prime}\left(\lambda_{2}(x)\right)\right) c_{002}+g_{2}^{\prime}\left(\lambda_{2}(x)\right)\left(-c_{110}+c_{101}+c_{011}\right)\right) \gamma_{2} .
\end{aligned}
$$


If we take the particular case $d=p_{2}-p_{1}$ we have $\gamma_{0}=0, \gamma_{1}=-1, \gamma_{2}=1$, and so

$$
\begin{aligned}
D_{d} f(x)=( & \left.g_{1}^{\prime}\left(\lambda_{1}(x)\right)+g_{2}^{\prime}\left(\lambda_{2}(x)\right)\right)\left(c_{101}-c_{110}\right) \\
& +\left(a-g_{2}^{\prime}\left(\lambda_{2}(x)\right)\right)\left(c_{002}-c_{011}\right)+\left(a-g_{1}^{\prime}\left(\lambda_{1}(x)\right)\right)\left(c_{011}-c_{020}\right) .
\end{aligned}
$$

Therefore if we let $c_{101}=1$ and $c_{\mathbf{i}}=0$ otherwise, then, since the control net of $f$ is increasing in the direction $p_{2}-p_{1}$ and $\left(\phi_{\mathbf{i}}\right)_{|\mathbf{i}|=2}$ is AMP, we deduce that

$$
g_{1}^{\prime}\left(\lambda_{1}(x)\right)+g_{2}^{\prime}\left(\lambda_{2}(x)\right)=D_{d} f(x) \geq 0 .
$$

If on the other hand we let $c_{002}=1$ and $c_{\mathbf{i}}=0$ otherwise, we find that we must have

$$
a-g_{2}^{\prime}\left(\lambda_{2}(x)\right) \geq 0 .
$$

By considering also the symmetric cases $d=p_{0}-p_{2}$ and $d=p_{1}-p_{0}$ one deduces the properties of $g_{0}, g_{1}, g_{2}$ in (ii).

For the sufficiency part, suppose that the system $\left(\phi_{\mathbf{i}}\right)_{|\mathbf{i}|=2}$ satisfies property (ii). Then if $d=p_{2}-p_{1}$, the directional derivative $D_{d} f(x)$ satisfies equation (5.4); and since the coefficients of the three differences are nonnegative we deduce that if $p$ is increasing in the direction $p_{2}-p_{1}$, then so is $f$. The same is true for the other two directions $p_{0}-p_{2}$ and $p_{1}-p_{0}$, and so the system $\left(\phi_{\mathbf{i}}\right)_{|\mathbf{i}|=2}$ is AMP.

Since we know that Bernstein polynomials are axially monotonicity preserving, they must satisfy property (ii) of Theorem 5.1. Indeed, if we let $a=1, b_{0}=b_{1}=$ $b_{2}=0$ and $g_{0}(s)=g_{1}(s)=g_{2}(s)=g(s)=s(1-s)$, we have that $g_{k}^{\prime}(s)=1-2 s \leq a$ and

$$
g^{\prime}(s)+g^{\prime}(t)=2(1-s-t) \geq 0
$$

when $s, t \geq 0$ and $s+t \leq 1$. So the conditions of property (ii) are satisfied. Moreover we find for example that, from (5.3), $\phi_{200}=\lambda_{0}-\lambda_{0}\left(1-\lambda_{0}\right)=\lambda_{0}^{2}$, and from (5.2),

$$
\begin{aligned}
\phi_{110} & =\lambda_{0}\left(1-\lambda_{0}\right)+\lambda_{1}\left(1-\lambda_{1}\right)-\lambda_{2}\left(1-\lambda_{2}\right) \\
& =\lambda_{0}\left(1-\lambda_{0}\right)+\lambda_{1}\left(1-\lambda_{2}\right)-\left(1-\lambda_{0}-\lambda_{1}\right)\left(\lambda_{0}+\lambda_{1}\right)=2 \lambda_{0} \lambda_{1},
\end{aligned}
$$

and indeed $\phi_{\mathbf{i}}=B_{\mathbf{i}}$ for all $\mathbf{i},|\mathbf{i}|=2$.

\section{MP AND SMP SYSTEMS WHEN $n=2$}

In the previous section we characterized all systems $\left(\phi_{\mathbf{i}}\right)_{|\mathbf{i}|=n}$ of six differentiable functions which are axially monotonicity preserving. In this section we deal with corresponding characterizations of monotonicity preserving systems and strongly monotonicity preserving systems when $n=2$. These results allow us to make direct comparisons between the three classes of systems in the case $n=2$.

Taking into account Theorem 5.1 and the following result, we see that AMP systems and MP systems are closely related in the case $n=2$.

Theorem 6.1. Let $\left(\phi_{\mathbf{i}}\right)_{|\mathbf{i}|=2}$ be a system of six differentiable functions. Then the following two properties are equivalent:

(i) $\left(\phi_{\mathbf{i}}\right)_{|\mathbf{i}|=2}$ is $M P$.

(ii) The functions $\phi_{\mathbf{i}}$ satisfy property (ii) of Theorem 5.1, and in addition

$$
g_{0}^{\prime}\left(\lambda_{0}\right)+g_{1}^{\prime}\left(\lambda_{1}\right)+g^{\prime}\left(\lambda_{2}\right) \geq a, \quad \lambda_{0}, \lambda_{1}, \lambda_{2} \geq 0, \quad \lambda_{0}+\lambda_{1}+\lambda_{2} \leq 1 .
$$


Proof. Suppose that $\left(\phi_{\mathbf{i}}\right)_{|\mathbf{i}|=2}$ is MP. Then since it is also AMP, property (ii) of Theorem 5.1 holds. It remains to demonstrate equation (6.1). Since $\left(\phi_{\mathbf{i}}\right)_{|\mathbf{i}|=2}$ is AMP, we know that for any $d \in \mathbb{R}^{2}$, the directional derivative of $f$ is given by equation (5.4), which by comparing the coefficients $c_{\mathbf{i}},|\mathbf{i}|=2$, can be rearranged into the form

$$
\begin{aligned}
D_{d} f(x)=\left(a-g_{0}^{\prime}\left(\lambda_{0}(x)\right)\right) E_{d} c_{\mathbf{e}_{0}}+\left(a-g_{1}^{\prime}\left(\lambda_{1}(x)\right)\right) E_{d} c_{\mathbf{e}_{1}}+\left(a-g_{2}^{\prime}\left(\lambda_{2}(x)\right)\right) E_{d} c_{\mathbf{e}_{2}} \\
+\left(g_{0}^{\prime}\left(\lambda_{0}(x)\right)+g_{1}^{\prime}\left(\lambda_{1}(x)\right)+g_{2}^{\prime}\left(\lambda_{2}(x)\right)-a\right) F_{d} c_{\mathbf{0}} .
\end{aligned}
$$

Now choosing $\gamma_{0}=\gamma_{1}=1 / 2, \gamma_{2}=-1$ and letting $c_{110}=1, c_{200}=c_{020}=-1$ and $c_{\mathbf{i}}=0$ otherwise, we find that

$$
E_{d} c_{\mathbf{e}_{0}}=E_{d} c_{\mathbf{e}_{1}}=E_{d} c_{\mathbf{e}_{2}}=0, \quad F_{d} c_{\mathbf{0}}=1,
$$

and therefore

$$
g_{0}^{\prime}\left(\lambda_{0}(x)\right)+g_{1}^{\prime}\left(\lambda_{1}(x)\right)+g_{2}^{\prime}\left(\lambda_{2}(x)\right)-a=D_{d} f(x) \geq 0 .
$$

If on the other hand the system $\left(\phi_{\mathbf{i}}\right)_{|\mathbf{i}|=2}$ satisfies property (ii), then since $D_{d} f$ has the form (6.2) it immediately follows that if $p$ is increasing in the direction $d$ then so is $f$, and so $\left(\phi_{\mathbf{i}}\right)_{|\mathbf{i}|=2}$ is MP.

As we observed in Section 2, Bernstein polynomials are strongly monotonicity preserving. In contrast to axially monotonicity preservation and monotonicity preservation, we shall show that strong monotonicity preservation places a much stronger restriction on the system of functions in question. In fact, the following result shows that in the case $n=2$, the six functions are constrained to be polynomials of degree at most two, just as in the Bernstein case.

Theorem 6.2. Let $\left(\phi_{\mathbf{i}}\right)_{|\mathbf{i}|=2}$ be a system of six twice differentiable functions. Then the following two properties are equivalent:

(i) $\left(\phi_{\mathbf{i}}\right)_{|\mathbf{i}|=2}$ is $S M P$.

(ii) The functions $\phi_{\mathbf{i}}$ satisfy property (ii) of Theorem 5.1, and in addition

$$
g_{k}(\lambda)=A \lambda^{2} / 2+B_{k} \lambda+C_{k}, \quad k=0,1,2,
$$

for some constants $A, B_{0}, B_{1}, B_{2}, C_{0}, C_{1}, C_{2}$ such that $A+B_{0}+B_{1}+B_{2}=a$.

Proof. Suppose that $\left(\phi_{\mathbf{i}}\right)_{|\mathbf{i}|=2}$ is SMP. Since it is then also AMP, property (ii) of Theorem 5.1 holds and moreover, for any $d \in \mathbb{R}^{2}$, the directional derivative of $f$ with respect to $d$ is given by equation (6.2). Choosing $\gamma_{0}=\gamma_{1}=1 / 2, \gamma_{2}=-1$ and letting $c_{110}=1, c_{200}=c_{020}=-1$ and $c_{\mathbf{i}}=0$ otherwise, one finds that

$$
E_{d} c_{\mathbf{e}_{0}}=E_{d} c_{\mathbf{e}_{1}}=E_{d} c_{\mathbf{e}_{2}}=0, \quad F_{d} c_{\mathbf{0}}=1,
$$

and therefore

$$
g_{0}^{\prime}\left(\lambda_{0}(x)\right)+g_{1}^{\prime}\left(\lambda_{1}(x)\right)+g_{2}^{\prime}\left(\lambda_{2}(x)\right)-a=D_{d} f(x)=0
$$

for all $x \in T$. Now by an argument identical to that used in Lemma 3.4, and noticing that $g_{0}^{\prime}, g_{1}^{\prime}, g_{2}^{\prime}$ are differentiable, we deduce that

$$
g_{k}^{\prime}(\lambda)=A \lambda+B_{k}, \quad k=0,1,2,
$$

for some constants $A, B_{0}, B_{1}, B_{2}$. Summing $g_{0}^{\prime}, g_{1}^{\prime}, g_{2}^{\prime}$ and using (6.5) and (6.4), we find that $A+B_{0}+B_{1}+B_{2}=a$. Integrating equations (6.5) yields equations (6.3), and we have established property (ii). 
Conversely, if the system $\left(\phi_{\mathbf{i}}\right)_{|\mathbf{i}|=2}$ satisfies property (ii), then on substituting equation (6.4) into equation (6.2) we have for any $d \in \mathbb{R}^{2}$ that

$$
D_{d} f(x)=\left(a-g_{0}^{\prime}\left(\lambda_{0}(x)\right)\right) E_{d} c_{\mathbf{e}_{0}}+\left(a-g_{1}^{\prime}\left(\lambda_{1}(x)\right)\right) E_{d} c_{\mathbf{e}_{1}}+\left(a-g_{2}^{\prime}\left(\lambda_{2}(x)\right)\right) E_{d} c_{\mathbf{e}_{2}} .
$$

Since $g_{k}^{\prime} \leq a$ for all $k=0,1,2$, it follows that $\left(\phi_{\mathbf{i}}\right)_{|\mathbf{i}|=2}$ is SMP.

Substituting the expressions (6.3) into (5.2) and (5.3) and recalling that $\lambda_{0}, \lambda_{1}, \lambda_{2}$ are linear and sum to one, we find that

$$
\phi_{\mathbf{i}}(x)=-A B_{\mathbf{i}}(x) / 2+u_{\mathbf{i}}(x), \quad|\mathbf{i}|=2,
$$

for some linear functions $u_{\mathbf{i}}$. Thus, provided $A$ is non-zero, each $\phi_{\mathbf{i}}$ is a quadratic polynomial and its nonlinear term is the quadratic Bernstein polynomial $B_{\mathbf{i}}$ multiplied by a scalar. In the Bernstein case, $A=-2$ and $u_{\mathbf{i}}=0$.

\section{REFERENCES}

[1] J. M. Carnicer, M. García-Esnaola, and J. M. Peña, Convexity of rational curves and total positivity, J. Comp. Appl. Math. 71 (1996), 365-382. MR 97k:65043

[2] J. M. Carnicer and J. M. Peña, Shape preserving representations and optimality of the Bernstein basis, Adv. Comput. Math. 1 (1993), 173-196. MR 94i:65138

[3] J. M. Carnicer and J. M. Peña, Monotonicity preserving representations, Curves and Surfaces in Geometric Design, II, P. J. Laurent, A. Le Méhauté and L. L. Schumaker, (eds.), AKPeters, Boston, 1994, pp. 83-90. MR 95g:65198

[4] J. M. Carnicer and J. M. Peña, Total positivity and optimal bases, Total Positivity and its Applications (M. Gasca and C.A. Micchelli, eds.), Kluwer Academic Press, Dordrecht, 1996, pp. 133-155. MR 97i:41012

[5] G. Farin, Curves and Surfaces for Computer Aided Geometric Design, Academic Press, Boston, 1988. MR 90c:65014

[6] R. T. Farouki and T. N. T. Goodman, On the optimal stability of the Bernstein basis, Math. Comp. 65 (1996), 1553-1566. MR 97a:65021]

[7] T. N. T. Goodman, Variation diminishing properties of Bernstein polynomials on triangles, J. Approx. Theory 50 (1987), 111-126. MR 88g:41006

[8] T. N. T. Goodman, Further variation diminishing properties of Bernstein polynomials on triangles, Constr. Approx. 3 (1987), 297-305. MR 88j:41022

[9] T. N. T. Goodman, Shape preserving representations, Mathematical Methods in Computer Aided Geometric Design T. Lyche and L.L. Schumaker (eds.), Academic Press, New York, 1989, pp. 333-351. MR 91a:65031

[10] J. Hoschek and D. Lasser, Fundamentals of Computer Aided Geometric Design, A. K. Peters, Wellesley, MA, 1993. MR 94i:65003

[11] S. Karlin, Total Positivity, Stanford University Press, Stanford, 1968. MR 37:5667

[12] T. Sauer, Multivariate Bernstein polynomials and convexity, Comput. Aided Geom. Design 8 (1991), 465-478. MR 93a:41012

Sintef Applied Mathematics, P.O. Box 124 Blindern, 0314 Oslo, NORWAY

E-mail address: mif@math.sintef.no

Departamento de Matemática Aplicada, Universidad de Zaragoza, Edificio de Matemáticas, Planta 1A, 50009 Zaragoza, SPAin

E-mail address: jmpena@posta.unizar.es 